\title{
High accurate analysis by experiment and simulation using Bayesian inference for corrugated cardboard
}

\author{
Masahiko SHIMAMURA*, Yoshitaka EZAWA ${ }^{* *}$, Yoshiaki TAMURA ${ }^{* * *}$, Satoru TAKASHIMIZU* \\ and Daisuke SATOU ${ }^{* * * *}$ \\ *Graduate School of Engineering, Toyo University \\ 2100 Kujirai, Kawagoe, Saitama 350-8585, Japan \\ E-mail: s46a01310013@toyo.jp \\ ** Center for Computational Mechanics Research, Toyo University \\ 2100 Kujirai, Kawagoe, Saitama 350-8585, Japan \\ ${ }^{* * *}$ Faculty of Information Sciences and Arts, Toyo University \\ 2100 Kujirai, Kawagoe, Saitama 350-8585, Japan \\ ${ }^{\star \star * *}$ Kanepackage Co., Ltd \\ 1095-15 Minamimine, Iruma, Saitama 358-0046, Japan
}

Received 2 February 2016

\begin{abstract}
Corrugated cardboards are used in many fields. The design of corrugated cardboard, however, is based on experimentations. The subject of this paper is developing the technique for high accurate analysis of corrugated cardboards. The corrugated cardboard is complicated structures and its property is unknown. Therefore, it is difficult to analyze this structure. We did bending tests of corrugated cardboard and its homogenized analysis using finite elements. We estimated its property by comparing both results. The experimentations include a lot of variation because the sample varies widely. Therefore model verification and validation is necessary. We used Bayesian inference for this purpose. In Bayesian inference, a priori probability is important. We compared three a priori probabilities. The first one is a uniform distribution which means no a priori information. The second one is a normal distribution which indicates a priori information about ambiguous data of the property. The third one is a normal distribution of which mean is the exact property. It is not realistic to use the third one. Numerical results show a uniform distribution is useful for estimating the property. The variance of Bayesian inference using a uniform distribution is wide, but the mean value becomes exact value quickly. The numerical results show the validity of Bayesian inference.
\end{abstract}

Key words : Corrugated cardboard, Fineite element analysis, Data assimilation, Bayesian inference, Equivalent analysis

\section{Introduction}

Products that use composite materials, such as corrugated cardboard, are used in a wide variety of fields. Therefore, the importance of conducting numerical analysis of composite materials is increasing. When conducting numerical analysis of a composite material structure with anisotropic characteristics, one must give sufficient consideration to the parameters of composite material characteristics; therefore, to conduct a valid numerical analysis, it is important in engineering to calculate physical properties that are unique to the structure. In this study, we approached these issues using corrugated cardboard consisting of composite materials. In recent years, the use of corrugated cardboard has expanded, such as in transportation and cushioning material, and accurate strength design is necessary. However, corrugated cardboard parameters in a numerical analysis are adjusted based on literature and the previous experiences of the designer. Corrugated cardboard is structured by having a wave-shaped corrugating medium sandwiched between two base papers called liners. Since its flute is quite dense, it takes much time and effort to model, and it is unpractical 
to run the usual finite element analysis as it requires too much cost and time to generate mesh for each flute. Therefore, numerical simulation of corrugated cardboards has not progressed. As such, to analyze subjects for which discretization is difficult with a finite element method due to its analysis domain regularly repeating with a microscopic structure as a unit, and its dense degree of repetition, a study that applies a homogenization technique is being conducted (Kawashima et al., 2000). Therefore, it is quite effective to apply a homogenization technique to corrugated cardboard with the complex and microscopic structure of multiple flute (Terada et al., 1995; Terada and Kikuchi, 2003). To estimate the material properties of corrugated cardboard, in this study, by incorporating the experimental values of a bending test into numerical analysis using the homogenization technique, we were able to fully utilize the experimental results, and established a method that efficiently obtained the material properties, which are unknown parameters, using the response surface from the results of these numerical analyses. By comparing the results of numerical analysis of material properties with experimental values, we were able to verify its validity. In addition, to obtain material properties, we used experimental values of corrugated cardboards; however, the quality of corrugated cardboard materials is not uniform, and thus the reliability of analytical results obtained for material properties is essential in conducting numerical analysis. As such, the methodology of Model Verification \&Validation (V\&V) (Alvin et al., 1998) that increases the reliability of analytical results against modeling errors and parameter uncertainties is gaining attention and Bayesian inference (Watanabe, 2012; Fukasawa and Sumiya, 2009; Nishino and Fujino, 2013) in particular, is considered to be effective. There is no example of obtaining material properties of corrugated cardboard and conducting accuracy validation with the Model V\&V. Therefore, we quantitatively evaluated uncertainties for material properties obtained using Bayesian inference, and examined the validity. Also In this paper, we conducted discussion on especially elastic modulus for material property.

\section{Corrugated cardboard test method}

\subsection{Test material}

Figure 1(a) shows the structure of the single corrugated cardboard used for the experiment. Table 1 shows the dimensions, and Figure 1(b) shows the load direction due to an indenter. In addition, in this study, we used B flute that consists of corrugating medium MC120 and liner LB180 that is compliant to JIS P 3902 and JIS P 3904, and the experiment was performed at a temperature of 11 degrees and humidity of $38 \%$.

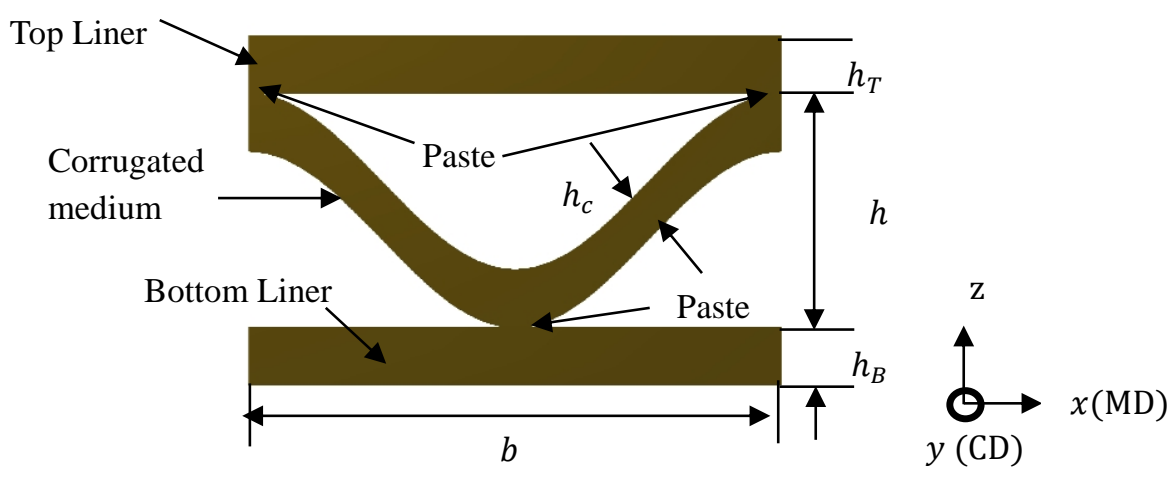

(a) Composition single corrugated cardboard

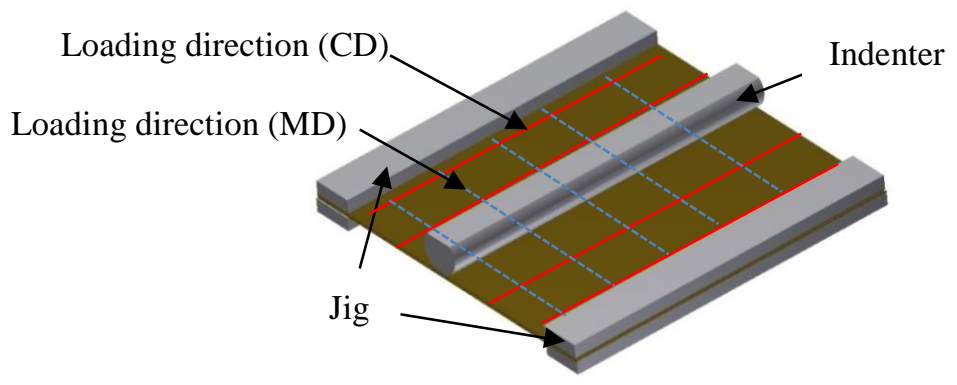

(b) Load direction of Indenter

Fig.1 Composition single corrugated cardboard and loading 
Table.1 Size of corrugated cardboard

\begin{tabular}{ccccc}
\hline \hline$h_{T}$ & $h_{B}$ & $h_{c}$ & $h$ & $b$ \\
\hline 0.3 & 0.3 & 0.3 & 2.4 & 6 \\
\hline & & & & $(\mathrm{mm})$
\end{tabular}

\subsection{Bending test}

To measure the flexural strength characteristics of corrugated cardboard, the sample was fixed with jigs and load was applied with an indenter as shown in Figure 2. At the speed of $10 \mathrm{~mm} / \mathrm{min}$, tests were conducted in the CD and MD ten times until the flute was completely crushed. The test fragment was to be changed in each experiment. Load and displacement characteristics of the test results are shown in Figure 3. It shows that with the maximum load, the bent part of the corrugated cardboard forms a line and buckling develops, confirming a rapid decrease in the load. Furthermore, when comparing each experimental result, there was a difference in the behavior of load and displacement, likely due to systematic errors.

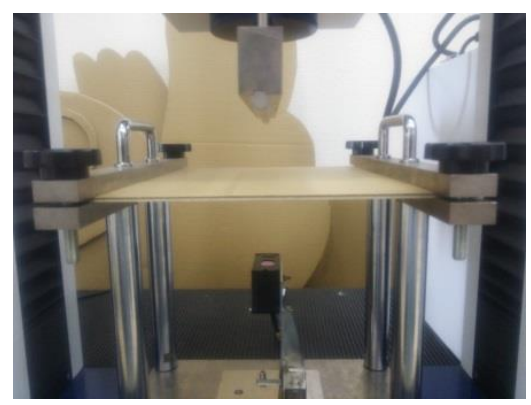

Fig.2 Bending test of corrugated cardboard

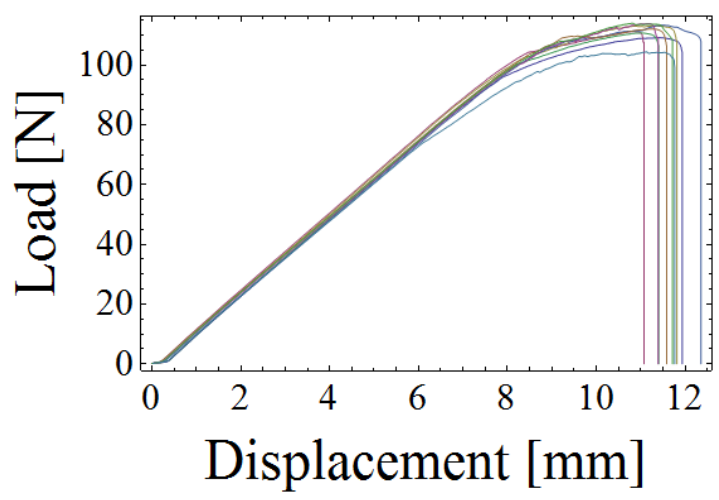

(a) $\mathrm{CD}$

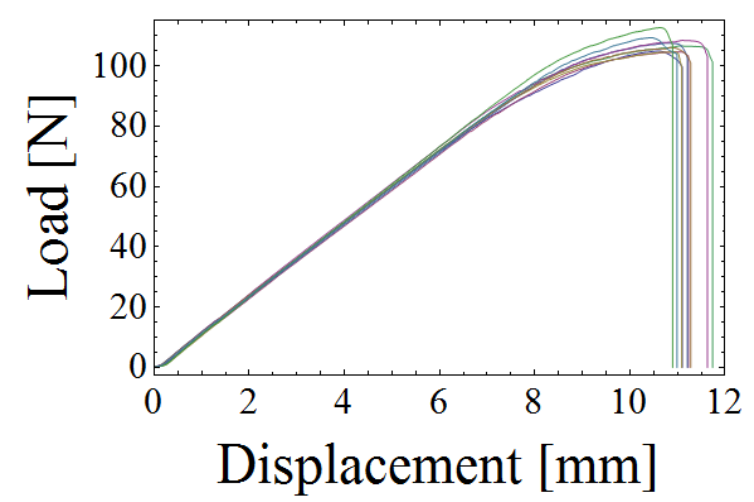

(b) MD

Fig3. Results of bending test

\section{Errors and the least square method}

\subsection{The regression line by the least square}

Data obtained from the experiment have errors as long as there are human factors involved. If a theoretical model is applied to such experimental values, one may use the least square method to determine the most appropriate function. If the model function is a primary function, a single regression model can be expressed as follows:

$f(x)=a x+b$

If $n$ data $\left(x_{1}, y_{1}\right),\left(x_{2}, y_{2}\right), \cdots,\left(x_{n}, y_{n}\right)$ is obtained: 
$\mathrm{a}=\frac{n \sum_{i=1}^{n} x_{i} y_{i}-\sum_{i=1}^{n} x_{i} \sum_{i=1}^{n} y_{i}}{n \sum_{i=1}^{n} x_{i}^{2}-\left(\sum_{i=1}^{n} x_{i}\right)^{2}}$

$\mathrm{b}=\frac{\sum_{i=1}^{n} x_{i}^{2} \sum_{i=1}^{n} y_{i}-\sum_{i=1}^{n} x_{i} y_{i} \sum_{i=1}^{n} x_{i}}{n \sum_{i=1}^{n} x_{i}^{2}-\left(\sum_{i=1}^{n} x_{i}\right)^{2}}$

By obtaining Equations (2) and (3), the slope of Equation (1) and the value for the intercept can be obtained. We used the results between displacement 0.5 and 2.0. These result shows linear response.

\subsection{Pretreatment for regression line}

The value of $b$ of equation (1) depends on the setting of the indenter. This value is not important for the analysis. Therefore, we set the value of $b$ at zero as in Equation (4) to recreate the behavior of experiment values in a numerical simulation. The analysis domain was limited to a linear range $(0.5 \leq$ displacement $\leq 2.0)$. Figure 4 shows regression lines of Equation (1) obtained from the least square method and Equation (4) in which the error due to the sample attachment is 0 , in both the CD and MD.

$y=a x$

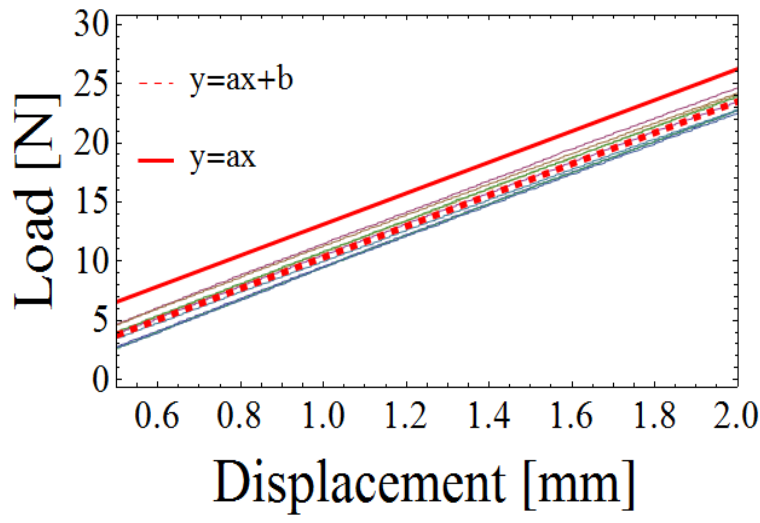

(a) $\mathrm{CD}$

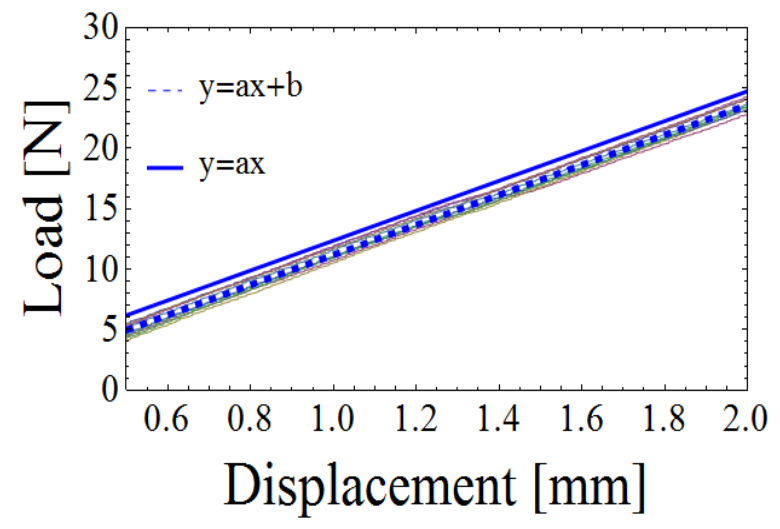

(b) MD

Fig.4 Analysis target area

\section{Numerical analysis of corrugated cardboard}

\subsection{The homogenization technique}

The homogenization technique (Kawashima et al., 2000) substitutes composite material with a microscopic periodic structure with an equivalent homogenous body and reflects the analysis of a microscopic basic structural unit (unit cell), consisting of one period of microscopic period structure, to evaluate macroscopic behavior. It is one of the effective averaging methods, and makes microscopic-macroscopic coupled behavior analysis possible.

If we set the coordinate system of a macro structure as $x$, and the microscopic coordinate system of the unit cell as $\mathrm{y}$, the following equation is established through microstructural parameter $\mu$ :

$y_{i}=\frac{x_{i}}{\mu}$

Assuming body force $\mathrm{f}$ that works in the domain $\Omega$ and surface force $\mathrm{t}$ that works in the domain $\Gamma_{t}$, the work of the virtual principle against the macro-model can be expressed as follows: 
$\int_{\Omega} D_{i j k l}^{\mu} \frac{\partial u_{k}^{\mu}}{\partial x_{i}} \frac{\partial v_{i}^{\mu}}{\partial x_{j}} d \Omega=\int_{\Omega} f_{i} v_{i}^{\mu} d \Omega+\int_{\Gamma_{t}} t_{i} v_{i}^{\mu} d \Gamma$

where $D_{i j k l}^{\mu}$ is the elastic tensor. Displacement $u^{\mu}$ of a structure consisting of a microscopic structure with periodicity is asymptotically expanded.

$u_{i}^{\mu}=u_{i}^{0}(x)+\mu u_{i}^{1}(x, y)+\cdots, y_{i}=\frac{x_{i}}{\mu}$,

where the following Equation can be established for Y-periodic function $\Psi(y)$ :

$\lim _{\mu \rightarrow 0} \int_{\Omega} \Psi\left(\frac{x}{\varepsilon}\right) d \Omega=\frac{1}{|Y|} \int_{\Omega} \int_{Y} \Psi(y) d Y d \Omega$

Here, $|Y|$ represents the volume of the unit cell. Equation (7) is substituted into Equation (6) and rearranged, and by applying Equation (8), it can be divided into Equation (10) that expresses microstructure and Equation (11) that expresses macrostructure.

$\frac{1}{\varepsilon^{2}} \int_{\Omega} D_{i j k l}^{\varepsilon} \frac{\partial u_{0 k}}{\partial y_{l}} \frac{\partial v_{i}}{\partial y_{j}} d \Omega=0$

$\frac{1}{\varepsilon} \int_{\Omega} D_{i j k l}^{\varepsilon}\left[\left(\frac{\partial u_{0 k}}{\partial x_{l}}+\frac{\partial u_{1 k}}{\partial y_{l}}\right) \frac{\partial v_{i}}{\partial y_{j}}+\frac{\partial u_{0 k}}{\partial y_{l}} \frac{\partial v_{i}}{\partial x_{j}}\right] d \Omega=0$

$\int_{\Omega} D_{i j k l}^{\varepsilon}\left[\left(\frac{\partial u_{0 k}}{\partial x_{l}}+\frac{\partial u_{1 k}}{\partial y_{l}}\right) \frac{\partial v_{i}}{\partial y_{j}}+\left(\frac{\partial u_{1 k}}{\partial x_{l}}+\frac{\partial u_{2 k}}{\partial y_{l}}\right) \frac{\partial v_{i}}{\partial y_{j}}\right] d \Omega=\int_{\Omega^{\varepsilon}} f_{i}^{\varepsilon} v_{i} d \Omega+\int_{\Gamma_{i}^{\varepsilon}} t_{i} v_{i} d \Gamma$

By applying divergence theorem and Equation (8) to Equation (9), the following Equation is obtained:

$u_{0}=u_{0}(x)$

where $u_{o}$ only depends on the overall structure. By applying Equation (8), Equation (12), and divergence theorem to Equation (10), and by introducing $\chi$ that satisfies Equation (9), Equation (14) is obtained:

$$
\begin{aligned}
& \int_{Y} D_{i j m n} \frac{\partial \chi_{m}^{k l}}{\partial y_{n}} \frac{\partial v_{1 i}}{\partial y_{j}} d Y=\int_{Y} D_{i k j l} \frac{\partial v_{1 i}}{\partial y_{j}} d Y \quad \forall v_{1} \in V_{Y} \\
& u_{1 i}=-\chi_{i}^{k l}(x, y) \frac{\partial u_{o k}(x)}{\partial x_{l}}
\end{aligned}
$$

By applying Equation (8), Equation (12), Equation (14), and divergence theorem to Equation (11), the virtual principle of the overall structure can be derived.

$$
\int_{\Omega} D_{i j k l}^{h} \frac{\partial u_{0 k}}{\partial y_{l}} \frac{\partial v_{0 i}}{\partial y_{j}} d \Omega=\int_{\Omega}\left(\frac{1}{|Y|} \int_{Y} f_{i} d Y\right) v_{0 i} d \Omega+\int_{\Gamma_{t}} t_{i} v_{0 i} d \Gamma
$$


where Equation (16) is an equation that obtains the equivalent elastic constant in macroscopic structure, and if defined as a homogenization elastic tensor $D_{i j k l}^{h}$, we obtain:

$D_{i j k l}^{h}=\frac{1}{|Y|} \int_{Y}\left(D_{i j k l}-D_{i j m n} \frac{\partial \chi_{m}^{k l}}{\partial y_{n}}\right) d Y$

In addition, displacement $u^{\varepsilon}$, strain $\epsilon^{\varepsilon}$, and stress $\sigma^{\varepsilon}$ that take microscopic structures into consideration can be obtained with the following Equation:

$u_{i}^{\varepsilon}=u_{0 i}(x)-\varepsilon \chi_{i}^{k l}(x, y) \frac{\partial_{u 0 k}(x)}{\partial x_{l}}+\cdots$

$\epsilon_{i j}^{\varepsilon}=\frac{\partial u_{0 i}}{\partial x_{j}}-\frac{\partial \chi_{i}^{k l}}{\partial y_{j}} \frac{\partial u_{0 k}}{\partial x_{l}}+\cdots$

$\sigma_{i j}^{\varepsilon}=\left(D_{i j k l}-D_{i j m n} \frac{\partial \chi_{m}^{k l}}{\partial y_{n}}\right) \frac{\partial u_{0 k}}{\partial x_{l}}+\cdots$

We used the finite element method calculating equation (13),(14),(15),(16),(17),(18) and (19).

\subsection{Microscopic structure finite element method model}

Since flute of corrugated cardboard have periodicity, we prepared a finite element model that uses one flute as a unit cell as shown in Figure 5.

To model corrugating medium, we approximated with Sine wave. Poison ratio was literature (Kawashima et al., 2000; Ishibuchi et al., 1994) reference and element decomposition was performed with appropriate number of nodes and elements considering the analysis time. Details are shown in Table 2.

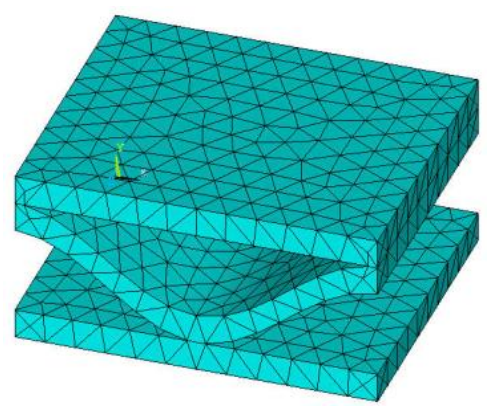

Fig.5 Unit cell finite element method for corrugated cardboard

Table. 2 Specifiction of unit cell finite element method

\begin{tabular}{|c|c|c|}
\hline \multicolumn{2}{|c|}{$\operatorname{Size}(\mathrm{mm})$} & $x=6.0, y=6.0, z=3.0$ \\
\hline \multicolumn{2}{|c|}{ Element Type } & Quadratic element \\
\hline \multicolumn{2}{|c|}{ Number of nodes } & 6099 \\
\hline \multicolumn{2}{|c|}{ Number of elements } & 2973 \\
\hline \multicolumn{2}{|c|}{ Number of materials } & 2 \\
\hline \multicolumn{2}{|c|}{ Material 1} & Liner \\
\hline \multicolumn{2}{|c|}{ Material 2} & Corrugated medium \\
\hline \multirow{2}{*}{ Poison ratio } & Liner & 0.6 \\
\hline & Corrugated medium & 0.3 \\
\hline
\end{tabular}




\subsection{Overall structure finite element method model}

We use the homogenized elastic stiffness obtained in Section4.2. We prepared a finite element method model that uses the test fragment used in the bending test as the overall structure. Figure 6 shows the finite element model, and Table 3 shows the details. Boundary conditions are shown in Figure 6(a): if analyzing in the CD, cross-directional xyz direction $C_{1}$ and $C_{2}$ were constant, and $-20 \mathrm{~N}$ was applied in the line z direction on the both $L_{1}$ and $L_{2}$; if analyzing in the MD, cross-directional xyz direction $C_{3}$ and $C_{4}$ were constant, and $-20 \mathrm{~N}$ was applied in the line $\mathrm{z}$ direction on the both $L_{3}$ and $L_{4}$.

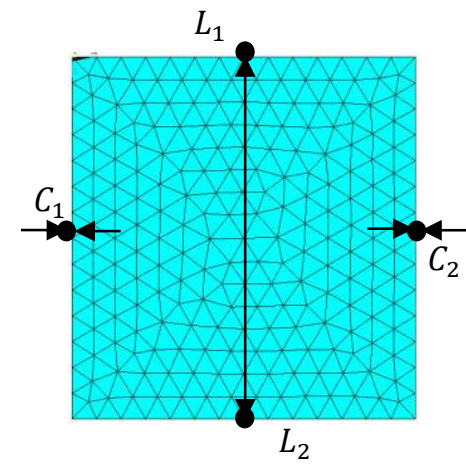

(a) $\mathrm{CD}$

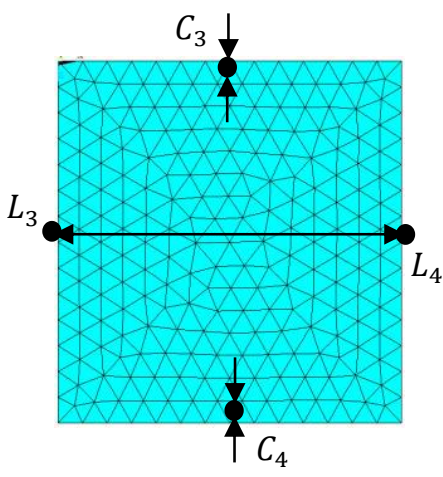

(b) MD

Fig.6 finite element method for corrugated cardboard for macro model

Table.3 Specifiction of finite element method for macro model

\begin{tabular}{c|c}
\hline \hline Size $(\mathrm{mm})$ & $\mathrm{X}=250, \mathrm{y}=250, \mathrm{z}=3.0$ \\
\hline Number of nodes & 3019 \\
\hline Number of elements & 1492 \\
\hline
\end{tabular}

\section{Method of calculating material constant}

\subsection{Formulation of elastic modulus search}

In this study, we targeted both the CD and MD of the corrugated cardboard that receives point load as an external force, and numerical simulation searched for elastic modulus that fit the displacement due to known $20 \mathrm{~N}$ load from the regression line using the homogenization technique. $e_{1}$ represents the liner, while $e_{2}$ represents the elastic modulus of the corrugating medium, respectively, and can be formulated as in Equation (20).

$\operatorname{im}\left(F_{20}\right)\left(\left\{\left(e_{1}, e_{2}\right) \mid e_{1}, e_{2} \in\{1000,2000,3000, \cdots, 10000\}\right\}\right)$

\subsection{Response surface model}

Response surface method is technique for continuous carved surface complementary discrete multiple solution, and predictive accuracy and that depend on approximate function and interpolation technique but it have estimating advantage relativity well-approximate equation by low sample number. If analysis point of $\operatorname{search} x_{i}(i=1,2, \cdots, n)$ and approximation error as $\varepsilon$, elastic modulus of liner and corrugated medium y can be expressed as equation (21).

$y=f\left(x_{1}, x_{2}, \cdots, x_{n}\right)+\varepsilon$

Figure (7) shows response surface of CD and MD obtained in this research. Point is result of numerical simulation and curved surface is response surface. There was difference in both numerical simulation and response surface from approximation error as $\varepsilon$. But obtained numerical simulation and response surface are consistent, and the number of sample used here was sufficient enough to give accurate response surface. 


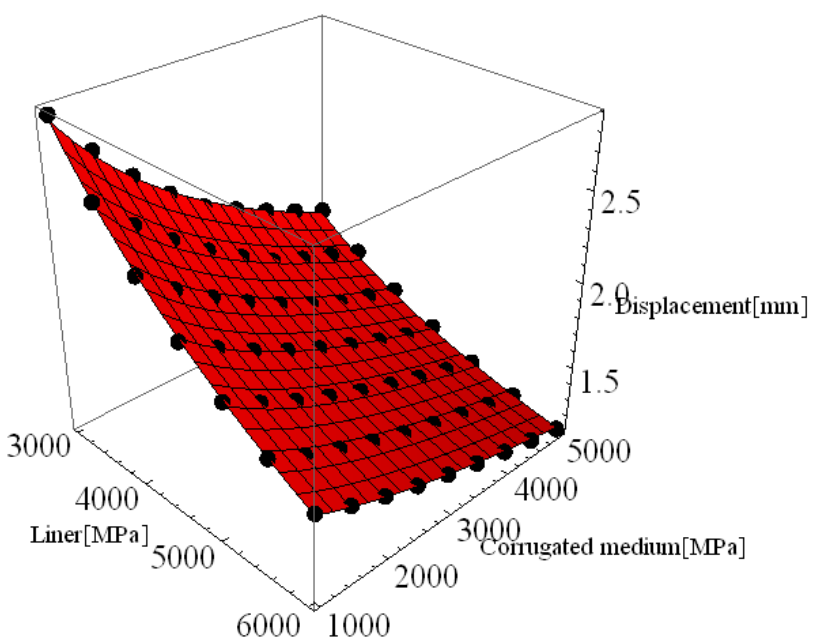

(a) $\mathrm{CD}$

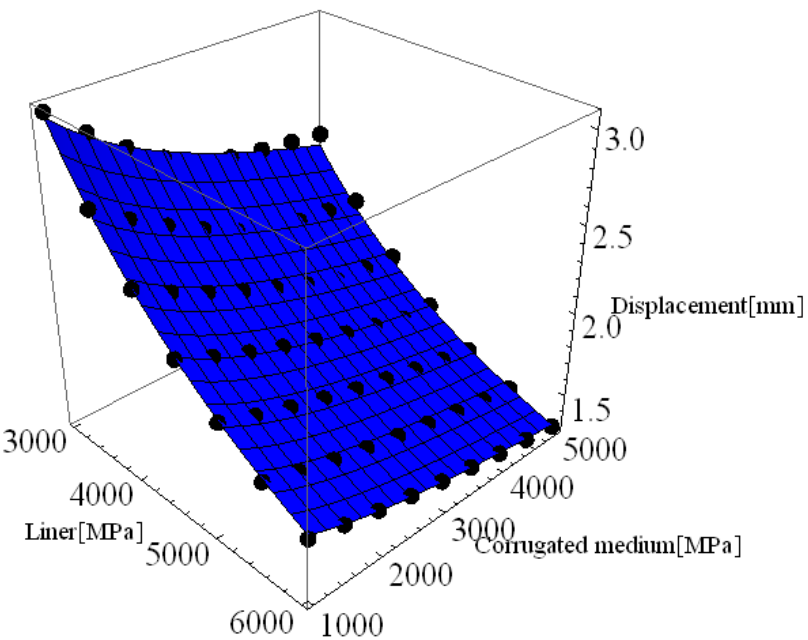

(b) $\mathrm{MD}$

Fig.7 Response surface model search for elastic modulus

\subsection{Calculation of material constant}

We considered the coordinates of CD and MD intersection as shows Figure (8) by Equation (22) in solution space of responses surface that the estimated elastic modulus of liner and corrugated medium.

$\left(e_{1}^{*}, e_{2}^{*}\right)=\underset{\left(e_{1}, e_{2}\right)}{\operatorname{argmin}}\left(r s^{\prime}\left(\text { data }^{\prime}\right)\left(e_{1}, e_{2}\right)-r s^{\prime \prime}\left(\text { data }^{\prime \prime}\right)\left(e_{1}, e_{2}\right)\right)^{2}$

Where $e_{1}^{*}$ and $e_{2}^{*}$ are the estimated elastic modulus of liner and corrugated medium, $r s^{\prime}$ and data' are response surface and data used in the response surface of $\mathrm{CD}, r s^{\prime \prime}$ and data" are response surface and data used in the response surface of MD. Therefore, $r s^{\prime}\left(\right.$ data $\left.^{\prime}\right)\left(e_{1}, e_{2}\right)$ and $r s^{\prime \prime}\left(\right.$ data" $\left.^{\prime \prime}\right)\left(e_{1}, e_{2}\right)$ are shows response surface of liner and corrugated medium of $\mathrm{CD}$ and $\mathrm{MD}$. In this study, we get intersection coordinate by calculating difference $r s^{\prime \prime}\left(\right.$ data" $\left.^{\prime \prime}\right)\left(e_{1}, e_{2}\right)$ from $r s^{\prime}\left(\right.$ data $\left.^{\prime}\right)\left(e_{1}, e_{2}\right)$ as a method of obtaining the coordinate of CD and MD intersection. Material properties of Liner and Corrugated medium calculated by intersection coordinate are shown in Table 4.

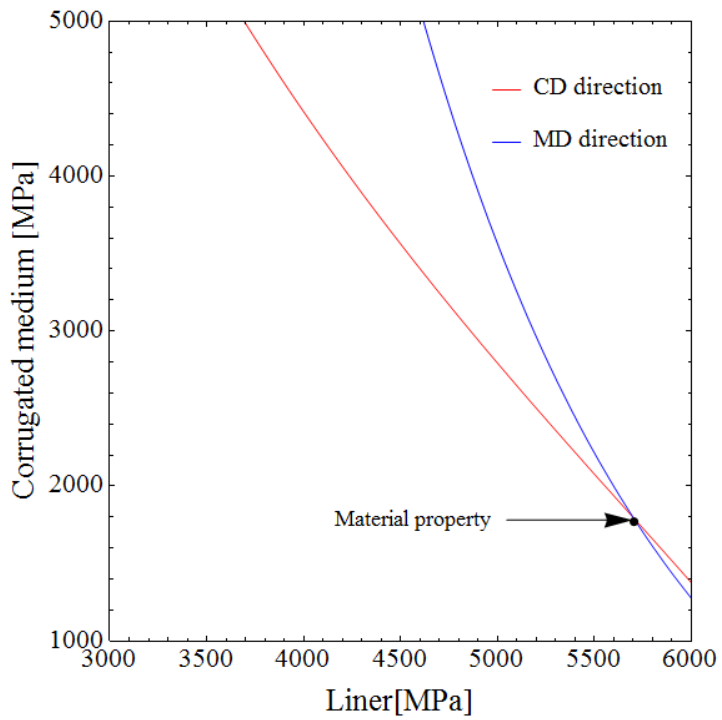

Fig.8 Inference of elastic modulus by intersection of coordinate 
Table4. elastic modulus by inference

\begin{tabular}{c|c|c}
\hline \hline & Liner & Corrugated medium \\
\hline $\begin{array}{c}\text { elastic modulus } \\
(\mathrm{MPa})\end{array}$ & 5700 & 1850 \\
\hline
\end{tabular}

\section{Verification of elastic modulus}

We will verify if the elastic modulus obtained in this research are valid. In the verification used elastic modulus both liner and corrugated medium of estimated Table 4 by this research. Figure 9 shows the results of comparing results of regression lines and numerical analysis by conducting an analysis under the condition of applying a load on a linear range using the homogenization technique as shown in Section 4. In this study, by rationally incorporating displacement data of the regression line into a numerical simulation, elastic modulus were obtained. Therefore, both in the $\mathrm{CD}$ and $\mathrm{MD}$, results are consistent with regression line values, confirming the validity of elastic modulus.

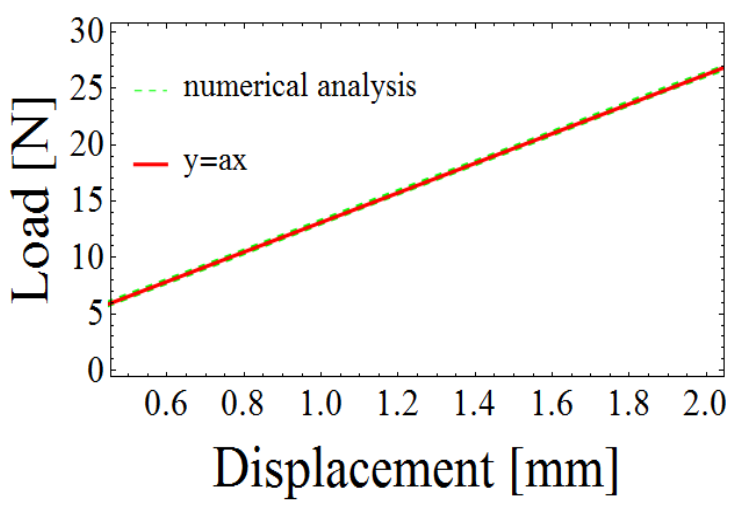

(a) CD

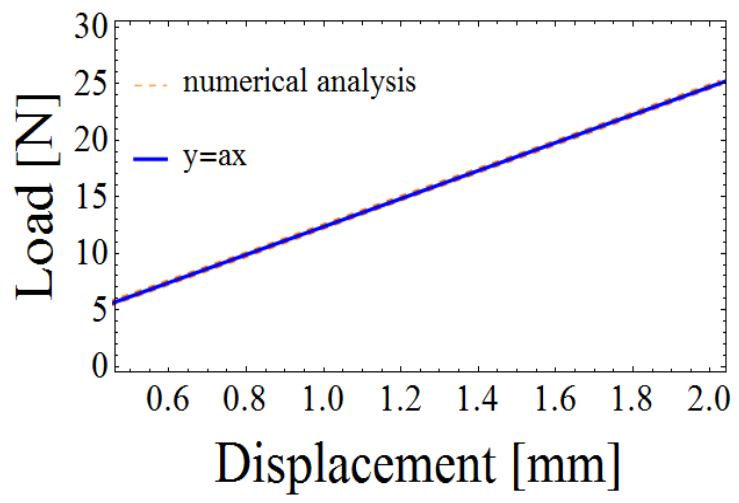

(b) MD

Fig.9 Comparison of between regression line and numerical analysis

\section{Reducing uncertainty}

\subsection{Posterior distribution estimation method}

Bayesian inference determines posterior distribution by uploading observational data to parameters of prior distribution, which consist of information already obtained and the prior knowledge of the analyst. Bayes' theorem can be expressed with Equation (23). Assuming the parameter the elastic modulus of liner and corrugated medium as $\theta$ and observational data as $d$, posterior distribution $p(\theta \mid d)$ can be obtained from the product of likelihood $f(d \mid \theta)$, which is probability of obtaining observation data as a condition for elastic modulus has particular value.

$p(\theta \mid d) \propto f(d \mid \theta) p(\theta)$

In other words, posterior distribution is proportional to prior distribution and likelihood, and can be considered to be the conditional probability of parameters when data were obtained. Since Bayesian inference can actively utilize prior distribution, even if the number of samples is low, it allows for estimation of unknown parameters that include uncertainties. In this study, we estimated parameter elastic modulus of liner and corrugated medium using Regression lines for observational data were obtained through the least square method against each measurement results in the CD and MD during the bending test.

\subsection{Likelihood}

We calculate probability distribution by using Bayesian inference. This method does not calculate fixed parameters unlike maximum likelihood method. Considering elastic modulus of liner and corrugated medium follows normal distribution of Equation(24), we estimate parameter $\mu, \sigma^{2}$ of elastic modulus by observed data $x$.

$N\left[x \mid \mu, \sigma^{2}\right]=\frac{1}{\sqrt{2 \pi} \sigma} \exp \left\{-\frac{\{x-\mu\}^{2}}{2 \sigma^{2}}\right\}$ 
where $\mu$ is average and $\sigma^{2}$ is variance. Therefore the likelihood for complete experimental data is Equation (25), (26).

$p\left(u^{\prime} \mid e_{1}, e_{2}, \mathrm{MD}\right)=\mathrm{N}\left[u^{\prime} \mid r s^{\prime}\left(\operatorname{data}^{\prime}\right)\left(e_{1}, e_{2}\right), \operatorname{var}\left(r s^{\prime}\left(\operatorname{data}^{\prime}\right)\left(e_{1}, e_{2}\right)\right)\right]$

$p\left(u^{\prime \prime} \mid e_{1}, e_{2}, \mathrm{CD}\right)=\mathrm{N}\left[u^{\prime \prime} \mid r s^{\prime \prime}\left(\operatorname{data}^{\prime \prime}\right)\left(e_{1}, e_{2}\right), \operatorname{var}\left(r s^{\prime \prime}\left(\operatorname{data}^{\prime \prime}\right)\left(e_{1}, e_{2}\right)\right)\right]$

where $\mathrm{u}$ is a displacement as observed data and var is a function for calculating the variance. In this study, we calculate posterior distribution, multiplying those likelihood by prior distribution.

\subsection{Setting prior distribution}

Prior distribution can reflect the analyst's subjective information. Details are shown in Table 5. In Case A, uniform distribution considers all parameters uniform when there is no prior knowledge, and in Case B, though it is unclear based on past experience, the range of model parameter values is roughly understood. Therefore, to reflect model parameter uncertainties as effectively as possible, we applied Gaussian distribution that has been through engineering study to this information. In Case C, we applied isotopic Gaussian distribution that reflects detailed information using the estimated model parameter values.

Table.5 Prior Distribution

\begin{tabular}{c|c|c|c|c}
\hline \hline & & Case A & Case B & Case C \\
\hline \multirow{2}{*}{ Average } & Liner & 5500 & 5500 & 5500 \\
\cline { 2 - 5 } & Corrugated medium & 1800 & 5500 & 1800 \\
\hline \multirow{2}{*}{ Variance } & Liner & $1.0 \times 10^{10}$ & 10000 & 10000 \\
\cline { 2 - 5 } & Corrugated medium & $1.0 \times 10^{10}$ & 10000 & 10000 \\
\hline
\end{tabular}

\subsection{Reducing uncertainty in posterior distribution}

In this study, to examine reduction of uncertainty, we made a ten-step observation where the total of two types of data (one in the $\mathrm{CD}$ and one in the MD) were represented as one step in order to make confirmation. Figure 10 shows the posterior distribution results of first step and final step. In addition, Table 5 shows mean and variance of ten steps. According to Figure 10 and table 5, in Cases A and B, with the number of data used in this research, it is difficult to say that uncertainty has been reduced to the point to guarantee the validity of elastic modulus. In Case B, results were biased toward prior distribution due to the impact of variance of prior distribution set for the estimated mean values. Thus, it was shown to be strongly influenced by prior distribution. In Case C, uncertainty was reduced even with a small number of data by reflecting more accurate prior information. Therefore, knowing more accurate prior information obviously contributes to gaining a valid value more quickly. In addition, while incomplete prior information has a negative impact on the estimation of mean value, in Case A without prior information, by using Bayesian inference, though reduction of uncertainty is slow, estimated mean value reaches a valid value quickly.

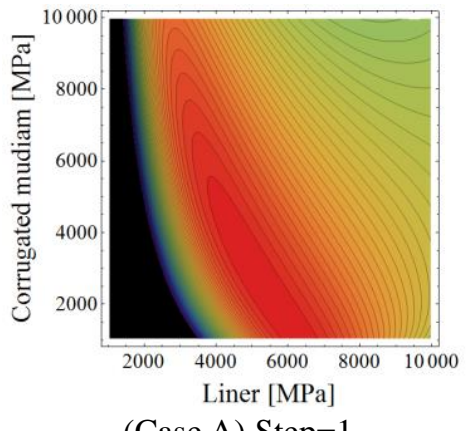

(Case A) Step=1

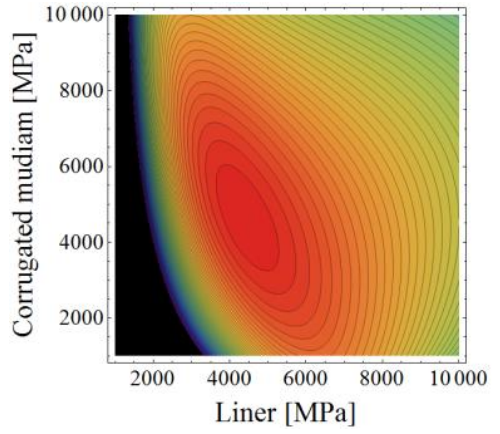

(Case B) Step=1

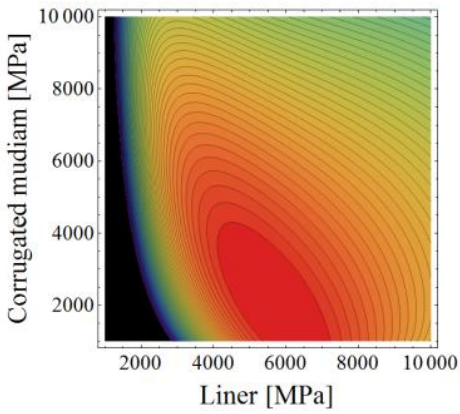

(Case C) Step=1 

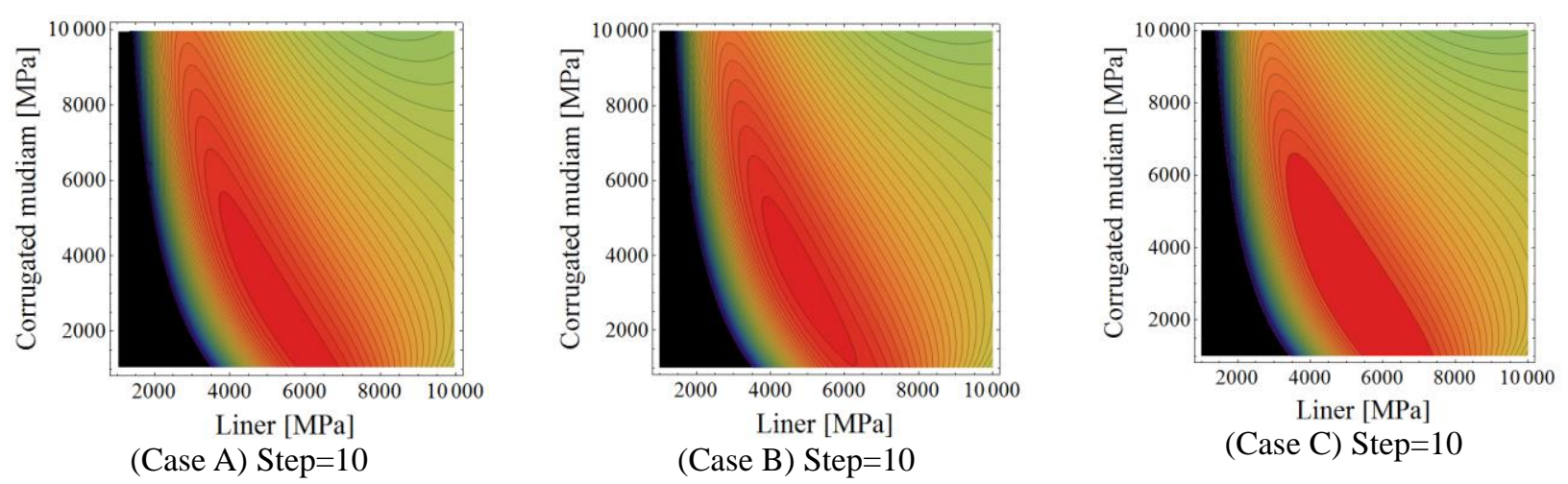

Fig.10 Posterior distribution

Table.5 Comparison of posterior distribution

\begin{tabular}{|c|c|c|c|c|}
\hline & & Case A & Case B & Case C \\
\hline \multirow{2}{*}{ Average } & Liner & 5494.9 & 4760.53 & 5629.13 \\
\hline & Corrugated medium & 2211.7 & 3491.87 & 2001.32 \\
\hline \multirow{2}{*}{ Variance } & Liner & 6892.2 & 1046.41 & 2492.13 \\
\hline & Corrugated medium & 16070 & 3342.29 & 5470.91 \\
\hline
\end{tabular}

\section{Conclusion}

In this study, we proposed a method of obtaining elastic modulus from a response surface prepared by using numerical analysis with a homogenization technique that focuses on the periodicity of corrugated cardboard and a bending test. Additionally, we then made a comparison with experimental values in order to confirm its validity. To reduce uncertainty in the obtained elastic modulus values, we conducted a quantitative evaluation using posterior distribution obtained using Bayesian estimation and conducted examination and validation. We present our findings below.

1. In a conventional finite element analysis, it took time and effort to prepare the model. In addition, since mesh is generated for each flute, the calculation cost is extremely high and inefficient. We focused on the periodicity of the corrugated cardboard, and used a flute of corrugated cardboard as a unit cell in order to conduct homogenization analysis of the test fragment as the overall structure. In this manner, by only preparing a model of the microstructure and simplified overall structure, and substituting the flute with a uniform material, analysis that takes microstructure into consideration was possible. This allows for a significant reduction in calculation cost, indicating that this is a useful method in analyzing corrugated cardboards.

2. We incorporate displacement of experimental value into numerical analysis of CD and MD, and created response surface by that result of analysis. Calculated material properties of liner and corrugated medium by the intersection of these response surface, displacement of numerical simulation analysis using calculated elastic modulus was compared with displacement of regression line. Result shows elastic modulus calculated by method proposed in this research is consistent with regression line values, confirming the validity of elastic modulus.

3. To reduce uncertainty of model parameters with Bayesian inference, posterior distribution was quantitatively examined. When more accurate prior distribution was used, even with a small number of samples, reduction in uncertainty was confirmed. However, with other prior distributions, such reduction was not confirmed. By using prior distribution for which analysts examined uncertainty, a valid posterior distribution was obtained. However, even when there is no prior information, by using Bayesian inference, though reduction of uncertainty is slow, estimated mean value reached a valid value quickly.

\section{References}

Alvin, K. F., et al., Uncertainty Quantification in Computational Structural Dynamics: a new paradigm for model validation, Society for Experimental Mechanics, Inc, 16th International Modal Analysis Conference, Vol. 2, (1998), pp.1191-1198.

Christopher, M. B., Pattern Recognition and Machine Learning (2006), pp.137-156, Springer.

Fukasawa, K. and Sumiya, T., Let's Start! Data Analysis with Bayesian Inference, Japanese Journal of Ecology, Vol. 59, (2009), pp.207-216 (in Japanese).

Hirohata, K., Kawakami, T., Mukai, M., Kawamura, N., Yu., Q. and Shirai, M., Proposal for Structural Reliability 
Design Method Based on the Response Surface Methodology and Bayes theory, Japan Society of Mechanical Engineers, Vol. 660, (2001), pp.1297-1304 (in Japanese).

Ishibuchi, H., Nagasawa, S., Yoshizawa, A. and Yoshitani, Y., Three-Dimensional Bending Stress Analysis for Corrugated Fibreboard Sheet, Japan Society of Mechanical Engineers, vol. 60, (1994), pp.2774-2781 (in Japanese).

Kawashima, Y., Nishimura, F. and Tezuka, A., Research on Structural Analysis of Paper Ware, Gifu Prefectural Research Institute of Manufacturing Information Technology Report, Vol. 2, (2000), pp.29-34 (in Japanese).

Nishio, M. and Fujino, Y., Baysian Inference Based Uncertainty Quantification and Calibration of Numerical Models of Existing Structures, JSCE A2 (Applied Mechanics), Vol. 69, (2013), pp.711-718 (in Japanese).

Steffen, L. L., Graphical Models (1996), pp.4-26, Oxford University Press Inc.

Terada, K. and Kikuchi, N., Introduction to Homogenization Technique (2003), Maruzen.

Terada, K., Yuge, K. and Kikuchi, N., Elasto-Plastic Analysis of Composite Materials Using the Homogenization Method, 1st Report, Formulation, Japan Society of Mechanical Engineers, Vol. 590, (1995), pp.2199-2205 (in Japanese).

Watanabe, S., Theory and Method of Bayesian Inference (2012), Corona Publishing Co. 